\title{
Distinct expression pattern and prognostic values of pituitary tumor transforming gene family genes in non-small cell lung cancer
}

\author{
SHAOLONG YANG ${ }^{1 *}$, XIAODI WANG ${ }^{2 *}$, JINGXING LIU $^{3 *}$, BISHA DING $^{4}$, \\ KAIRI SHI ${ }^{5}$, JING CHEN ${ }^{6}$ and WEIYANG LOU ${ }^{4}$
}

\begin{abstract}
${ }^{1}$ Department of Pathology, Zhengzhou Railway Vocational and Technical College, Zhengzhou, Henan 451460;
${ }^{2}$ Department of Emergency Medicine, The First Affiliated Hospital of Zhengzhou University, Zhengzhou, Henan 450000;

${ }^{3}$ Department of Intensive Care Unit, Changxing People's Hospital of Zhejiang, Huzhou, Zhejiang 313100;

${ }^{4}$ Department of Surgery, Program of Innovative Cancer Therapeutics, Division of Hepatobiliary and Pancreatic Surgery,

First Affiliated Hospital, College of Medicine, Zhejiang University, Key Laboratory of Combined Multi-Organ

Transplantation, Ministry of Public Health, Key Laboratory of Organ Transplantation, Hangzhou, Zhejiang 310003;

${ }^{5}$ Department of Orthopedics and Traumatology, Traditional Chinese Medicine Hospital of Cixi, Ningbo, Zhejiang 315300;

${ }^{6}$ Department of Oncology, First Affiliated Hospital of Jiaxing University, Jiaxing, Zhejiang 314000, P.R. China
\end{abstract}

Received December 12, 2018; Accepted July 5, 2019

DOI: $10.3892 / \mathrm{ol} .2019 .10844$

\begin{abstract}
Members of the pituitary tumor transforming gene (PTTG) family, including PTTG1, PTTG2 and PTTG3P, exhibit pivotal roles in the onset and progression of certain types of human cancer. However, to the best of our knowledge, a systematic study regarding the expression pattern and the prognostic values of PTTG family genes in non-small
\end{abstract}

Correspondence to: Dr Weiyang Lou, Department of Surgery, Program of Innovative Cancer Therapeutics, Division of Hepatobiliary and Pancreatic Surgery, First Affiliated Hospital, College of Medicine, Zhejiang University, Key Laboratory of Combined Multi-Organ Transplantation, Ministry of Public Health, Key Laboratory of Organ Transplantation, 79 Qingchun Road, Hangzhou, Zhejiang 310003, P.R. China

E-mail: 15968783311@163.com

Miss Jing Chen, Department of Oncology, First Affiliated Hospital of Jiaxing University, 1882 Central South Road, Jiaxing, Zhejiang 314000, P.R. China

E-mail: 344299731@qq.com

*Contributed equally

Abbreviations: NSCLC, non-small cell lung cancer; LUAD, lung adenocarcinoma; LUSC, lung squamous cell carcinoma; PTTG, pituitary tumor transforming gene; GEPIA, Gene Expression Profiling Interactive Analysis; TCGA, The Cancer Genome Atlas; FC, fold-change; OS, overall survival; HR, hazard ratio; CI, confidence interval

Key words: pituitary tumor transforming gene, non-small cell lung cancer, Kaplan-Meier plotter, prognosis, in silico analysis, Oncomine, UALCAN, gene expression profiling interactive analysis cell lung cancer (NSCLC) remains to be performed. The expression levels of PTTG family genes in NSCLC were successively determined using the Gene Expression Profiling Interactive Analysis, UALCAN and Oncomine databases. Subsequently, the Kaplan-Meier plotter database was used to assess the prognostic value of the PTTG family genes in patients with NSCLC, and to determine the associations between PTTG expression levels and the prognosis of patients based on different clinicopathological features, including cancer stage, grade, chemotherapy, radiotherapy, lymph node status, smoking history, and sex. PTTG1 was identified to be significantly upregulated in NSCLC in all three databases, whereas PTTG2 and PTTG3P were significantly upregulated in NSCLC in only the UALCAN database. Patients with NSCLC with higher expression levels of the three PTTG genes demonstrated shorter overall survival times. In summary, the results of the present study suggested that increased expression of PTTG family genes may serve as promising prognostic biomarkers for patients with NSCLC.

\section{Introduction}

Lung cancer is the leading cause of cancer-associated mortality worldwide in men and women, and its prognosis remains dismal with a five-year survival rate of $<15 \%$ (1). Non-small cell lung cancer (NSCLC), including lung adenocarcinoma (LUAD) and lung squamous cell carcinoma (LUSC), accounts for $\sim 75-80 \%$ of all lung cancer cases (2). In addition, although there are several treatment methods for patients with early-stage NSCLC, including surgery, chemotherapy, radiotherapy and molecular targeted therapy, the number of NSCLC cases is still increasing. In addition, treatment options for patients with advanced disease are limited (3), and almost $80 \%$ of patients with NSCLC are first diagnosed at an advanced stage (4). Therefore, there is an 
urgent requirement to conduct further investigations to study the mechanisms of the onset and progression of NSCLC, as well as to identify potential prognostic biomarkers. The development of prognostic biomarkers may improve the therapeutic choice for patients with NSCLC, and ultimately improve their prognosis.

The pituitary tumor transforming gene (PTTG) family is a novel class of homologous genes, which consists of three genes: PTTG1, PTTG2 and PTTG3P (5). The expression of PTTG1 is significantly upregulated in numerous endocrine-associated tumors, including pituitary, thyroid, breast and ovarian tumors (6). The dysregulation of PTTG1 enhances tumor cell proliferation, invasion and metastasis, and suppresses apoptosis (7-9). A number of studies have demonstrated that PTTG1 is an oncogene, and is overexpressed in human lung cancer. For example, Li et al (10) have reported that PPTG1 promotes the migration and invasion of NSCLC. In addition, Li et al (11) have demonstrated that knockdown of PTTG1 suppresses growth and invasion of LUAD. PTTG2 and PTTG3P, which are homologous genes of PTTG1, have recently been identified (5). Although little is understood regarding their biological functions, PTTG2 and PTTG3P have been revealed to be closely associated with the development of human cancer types. For example, Guo et al (12) have demonstrated that PTTG2 expression is significantly upregulated in glioblastoma, and its overexpression promoted glioblastoma cell proliferation and invasion. Weng et al (13) have demonstrated that PTTG3P enhances the in vitro proliferation and invasion of gastric cancer, and is an indicator of poor prognosis. However, to date, systematic analyses have not been performed for the mRNA expression pattern and prognostic roles of the PTTG family in NSCLC.

The present study determined the mRNA expression pattern of PTTG family genes in NSCLC, including LUAD and LUSC, using the Gene Expression Profiling Interactive Analysis (GEPIA), UALCAN and Oncomine databases. Subsequently, the prognostic values of PTTG family genes in NSCLC were assessed using the Kaplan-Meier plotter database. The Kaplan-Meier plotter database was also used to analyze the associations of PTTG1, PTTG2 and PTTG3P expression with the prognosis of patients based on clinicopathological features, including subtype, clinical stage, pathological grade, chemotherapy, radiotherapy, lymph node status, smoking history and sex. The in silico analysis performed in the present study may assist with the development of effective therapeutic targets and contribute to the improvement of the prognosis of patients with NSCLC.

\section{Materials and methods}

GEPIA database (http://gepia.cancer-pku.cn/detail.php). The expression levels of PTTG family genes in patients with LUAD and LUSC were evaluated using the GEPIA database, which is a newly developed interactive web server for analyzing the RNA sequencing expression data of 9,736 tumors and 8,587 normal samples from The Cancer Genome Atlas (TCGA) and Genotype-Tissue Expression projects (14). The results of differential expression analyses (PTTG1, PTTG2 and PTTG3P in LUAD/LUSC) are available on the website (GEPIA). Fold-change $(\mathrm{FC})>2$ and $\mathrm{P}<0.05$ were set as the thresholds of gene upregulation.
UALCAN database (http://ualcan.path.uab.edu/index.html). The expression levels of PTTG family genes in patients with LUAD and LUSC were further analyzed using the UALCAN database. UALCAN is a user-friendly, interactive web resource for analyzing TCGA transcriptome data (15). The analytical results were presented on the webpage (UALCAN). $\mathrm{P}<0.05$ was considered to indicate a statistically significant result.

Oncomine database (https://www.oncomine.org). Oncomine, which is a cancer microarray database and a web-based data-mining platform, was used to analyze the expression levels of PTTG family genes in LUAD and LUSC samples compared with normal lung samples using the differential expression analysis provided by the database $(16,17)$. FC $>1.5$, $\mathrm{P}<0.05$ and a gene rank in the top $10 \%$ were set as the thresholds for selecting the datasets.

Kaplan-Meier plotter database (http://kmplot.com/analysis). The prognostic value of the mRNA expression levels of PTTG family genes in patients with NSCLC was assessed using the online database Kaplan-Meier plotter, as previously described (18-20). Kaplan-Meier plotter was established using gene expression data and the survival information of patients with cancer downloaded from the Gene Expression Omnibus database (21). In the present study, the associations between PTTG1, PTTG2 and PTTG3P expression levels and the overall survival (OS) of patients with NSCLC were evaluated. Briefly, the three genes were first put into the database to obtain Kaplan-Meier survival plots. According to the median expression level, the cases were generally classified into low- and high-expression groups. A log-rank P-value, hazard ratio (HR) and $95 \%$ confidence interval (CI) were automatically calculated and presented on the webpage (Kaplan-Meier plotter). A log-rank $\mathrm{P}<0.05$ was considered to indicate a statistically significant difference.

\section{Results}

Expression levels of the PTTG family genes in patients with NSCLC. To investigate the mRNA expression levels of PTTG family genes in human NSCLC, three online databases, including GEPIA, UALCAN and Oncomine, were successively used. The GEPIA database was used to compare the mRNA expression levels of PTTG family genes in NSCLC samples with those in normal lung samples. PTTG1 expression was significantly upregulated in NSCLC subtypes LUAD and LUSC compared with normal lung samples (Fig. 1A). However, no significant differences were identified between PTTG2 or PTTG3P expression in cancer tissues and normal tissues (Fig. 1B and C). PTTG3P expression levels in LUAD, LUSC and corresponding normal controls were extremely low. Similar results of the expression levels of PTTG family genes in NSCLC were obtained using the UALCAN database (Fig. 1D-I). The UALCAN database demonstrated that the expression levels of PTTG2 and PTTG3P were low (transcripts per million $<1$ ), which may lead to inaccurate statistical differences of PTTG2 and PTTG3P.

Oncomine analysis was used to further evaluate the mRNA expression of PTTG family genes in NSCLC. Similar to the previous results, PTTG1 expression was significantly 
A
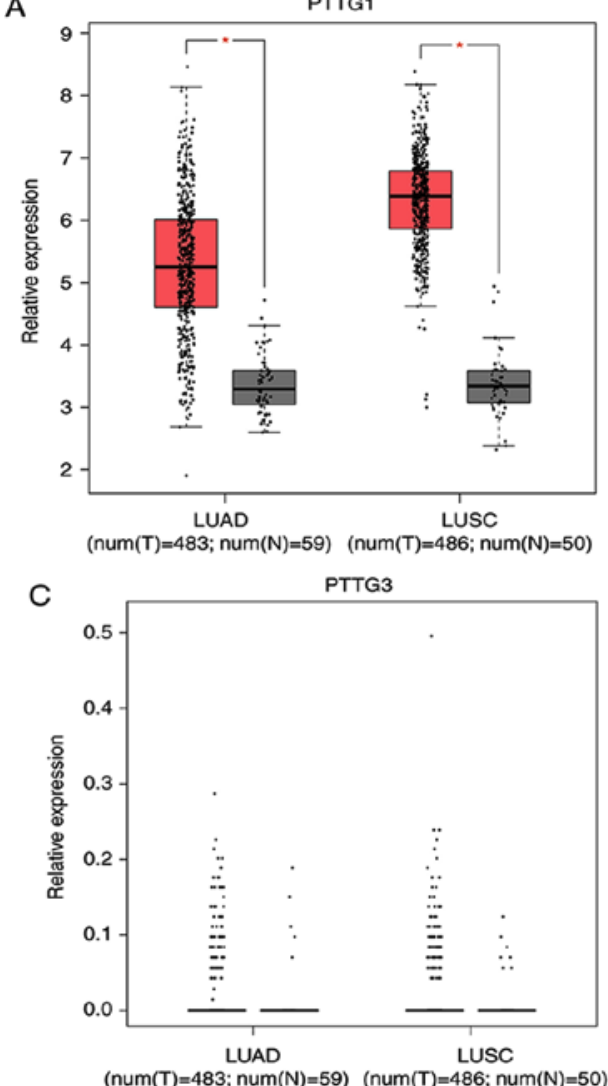

E Expression of PTTG2 in LUAD based on major cancer stages

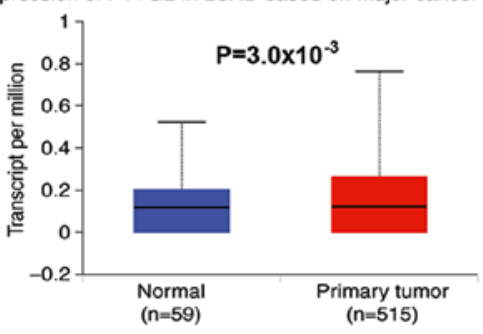

TCGA samples

G Expression of PTTG1 in LUSC based on major cancer stages

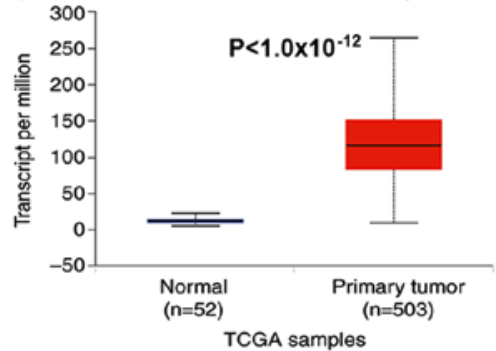

B

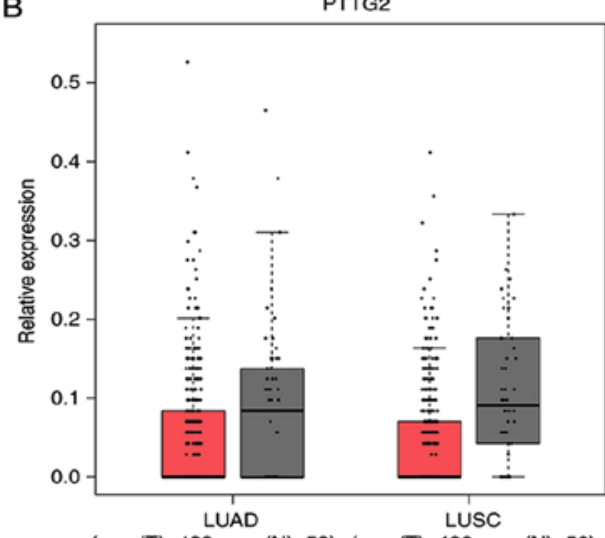

(num $(T)=483 ; \operatorname{num}(N)=59) \quad($ num $(T)=486 ; \operatorname{num}(N)=50)$

D Expression of PTTG1 in LUAD based on major cancer stages

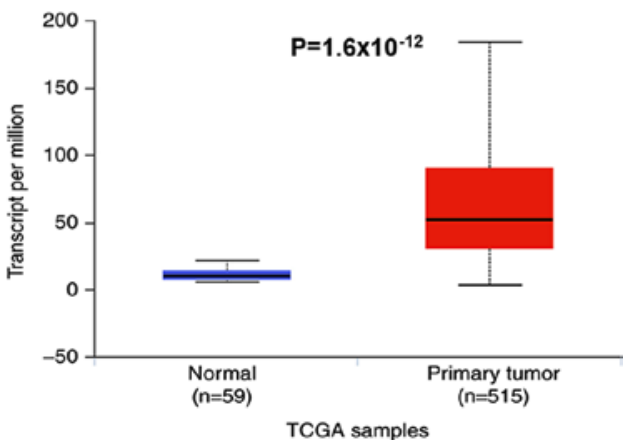

F Expression of PTTG3P in LUAD based on major cancer stages

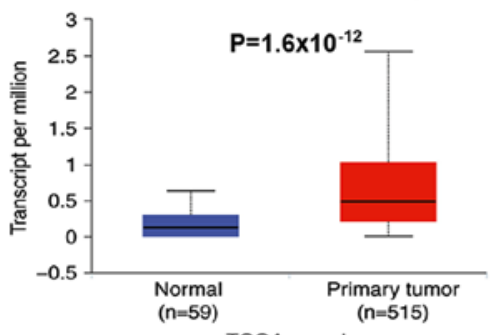

TCGA samples

H Expression of PTTG2 in LUSC based on major cancer stages

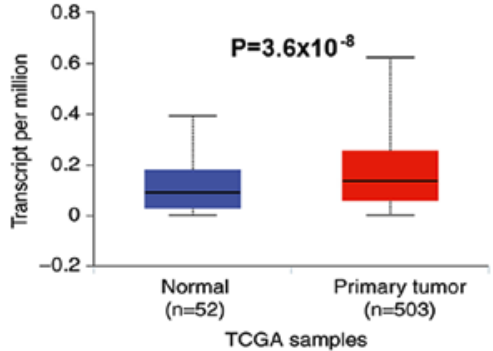

I Expression of PTTG3P in LUSC based on major cancer stages

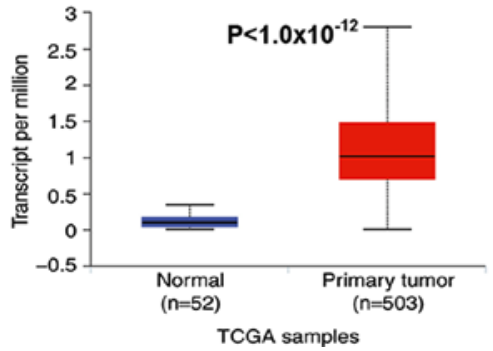

Figure 1. Expression of PTTG family in patients with non-small cell lung cancer from the GEPIA and UALCAN databases. (A-C) Expression of (A) PTTG1, (B) PTTG2 and (C) PTTG3P in patients with LUAD and LUSC in the GEPIA database. (D-F) Expression of (D) PTTG1, (E) PTTG2 and (F) PTTG3P in patients with LUAD in the UALCAN database. (G-I) Expression of (G) PTTG1, (H) PTTG2 and (I) PTTG3P in patients with LUSC in the UALCAN database. PTTG, pituitary tumor transforming gene; LUAD, lung adenocarcinoma; LUSC, lung squamous cell carcinoma; TCGA, The Cancer Genome Atlas. 
A

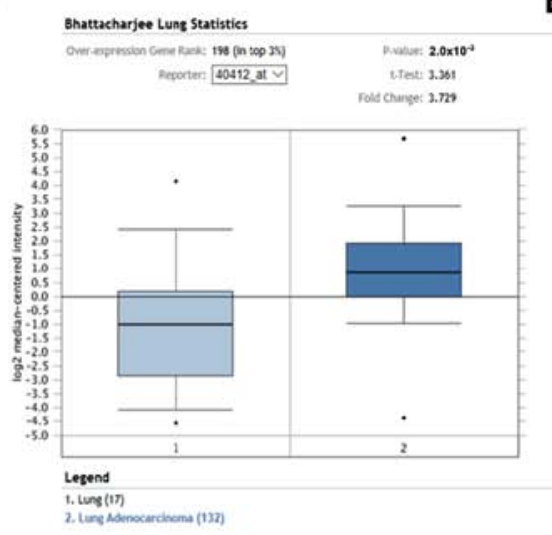

D

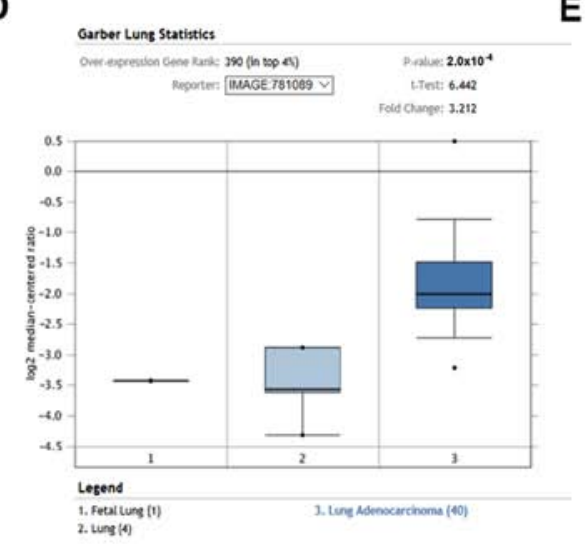

G

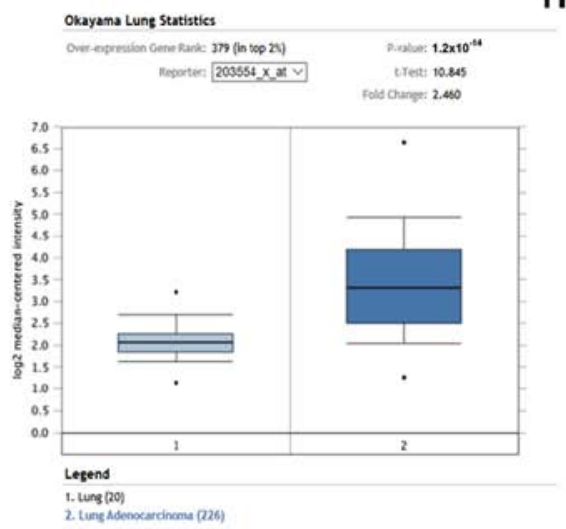

B

H

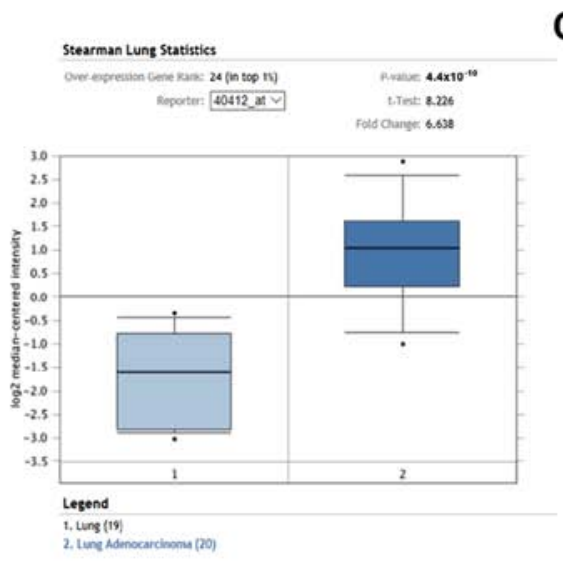

E
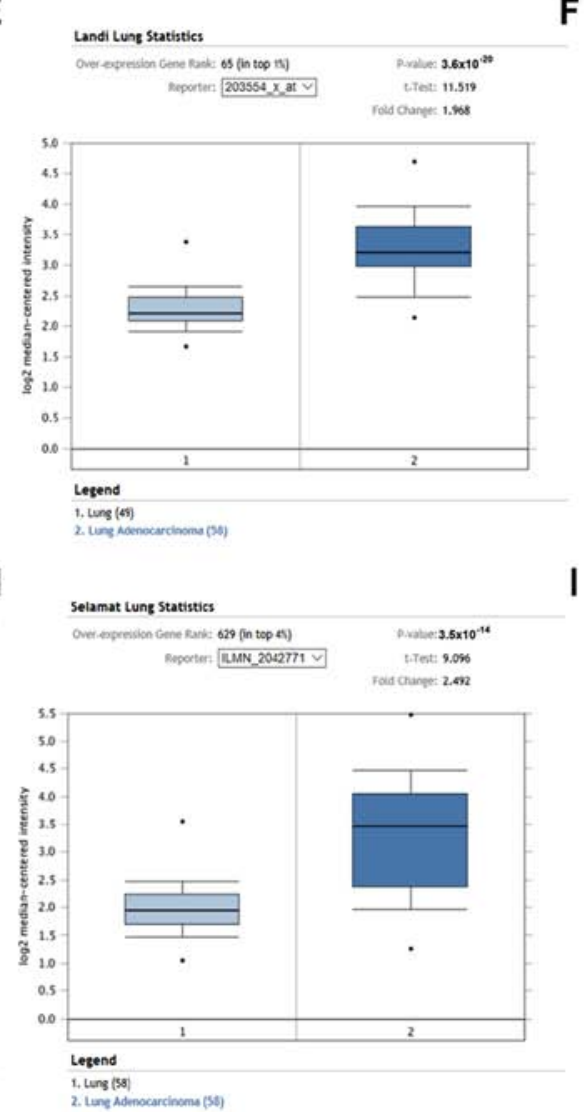

C
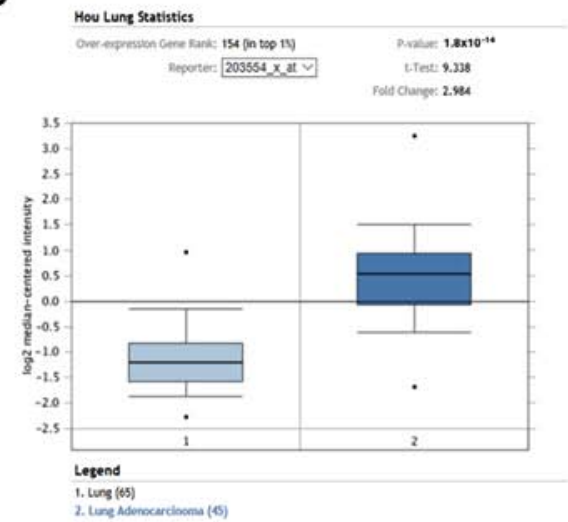

F
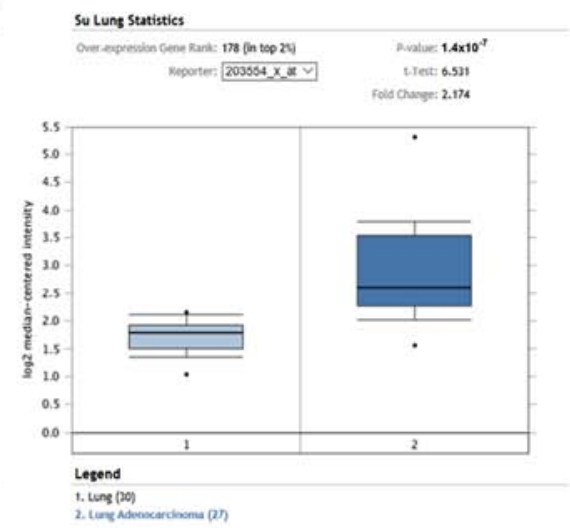

I

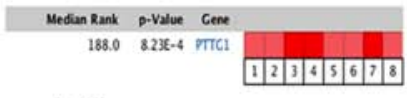

Legent

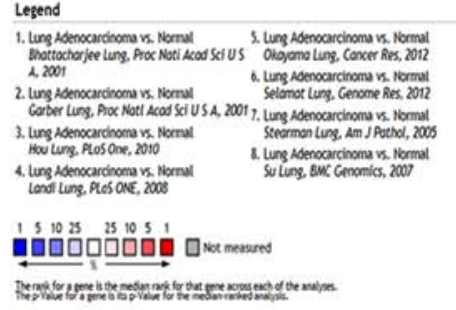

Figure 2. Meta-analyses of PTTG1 expression in patients with LUAD. PTTG1 expression in patients with LUAD according to different datasets from the Oncomine database: (A) Bhattacharjee lung dataset; (B) Stearman lung dataset; (C) Hou lung dataset; (D) Garber lung dataset; (E) Landi lung dataset; (F) Su lung dataset; (G) Okayama lung dataset and (H) Selamat lung dataset. (I) The details of the meta-analyses of PTTG1 in LUAD patients. The legends below the results contain detailed information on the selected datasets. PTTG, pituitary tumor transforming gene; LUAD, lung adenocarcinoma.

increased in both LUAD (Fig. 2) and LUSC (Fig. 3) compared with normal lung tissues. No significant differences were identified in the PTTG2 and PTTG3P expression levels between NSCLC and normal samples. The detailed information of the datasets with statistical significance information is presented in Table I. These data suggested that PTTG1 may be upregulated in NSCLC compared with normal lungs, whereas PTTG2 and PTTG3P are not dysregulated in NSCLC.

Prognostic values of PTTG family gene mRNA expression levels in patients with NSCLC. Kaplan-Meier plotter database was used to assess the effects of the mRNA expression levels of PTTG family genes on the survival of patients with NSCLC. The prognostic value of PTTG1 in all patients with NSCLC, patients with LUAD and patients with LUSC is presented in Fig. 4. Patients with NSCLC with high expression of PTTG1 exhibited significantly shorter OS time compared with patients with a low expression of PTTG1 (HR, 1.66; 95\% CI, 1.46-1.89; log-rank $\mathrm{P}=5.7 \times 10^{-15}$; Fig. 4A). High expression of PTTG1 in patients with LUAD indicated a poor prognosis (HR, 2.36; 95\% CI, 1.85-3.02; $\log$-rank $\mathrm{P}=1.9 \times 10^{-12}$; Fig. 4B). However, in patients with LUSC, high PTTG1 expression was not associated with OS (Fig. 4C).

The associations between PTTG2 mRNA expression levels and the overall survival of all patients with NSCLC, patients with LUAD and patients with LUSC were also analyzed. High PTTG2 expression levels were only significantly associated 
A Bhattacharjee lung statistics

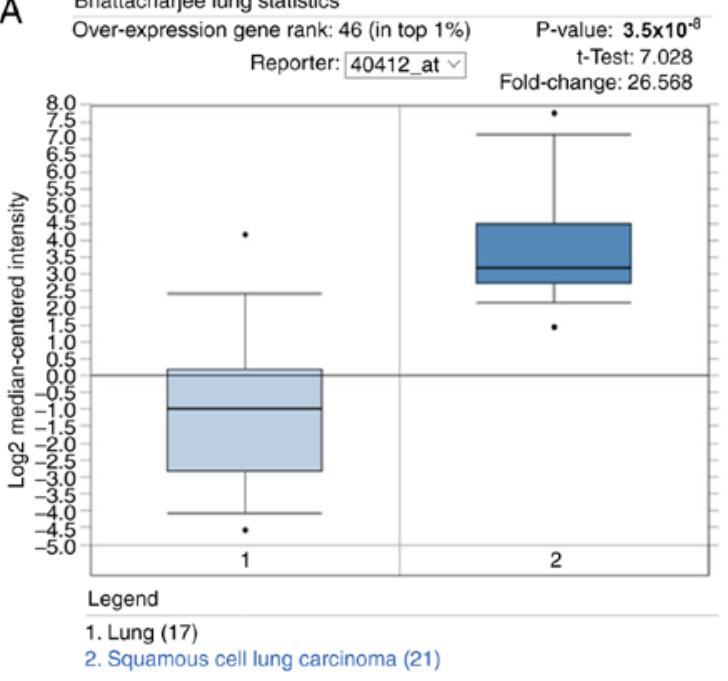

C Garber lung statistics
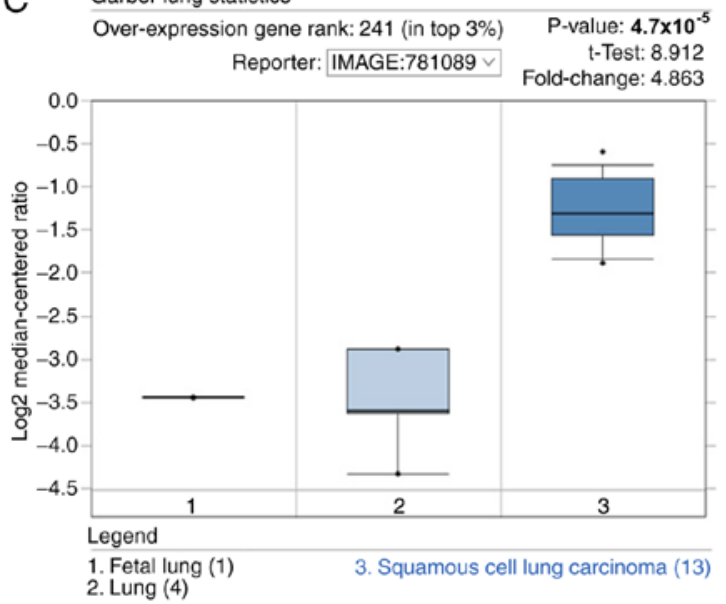

E Talbot lung statistics Over-expression gene rank: 817 (in top 10\%) P-value: $1.1 \times 10^{-6}$
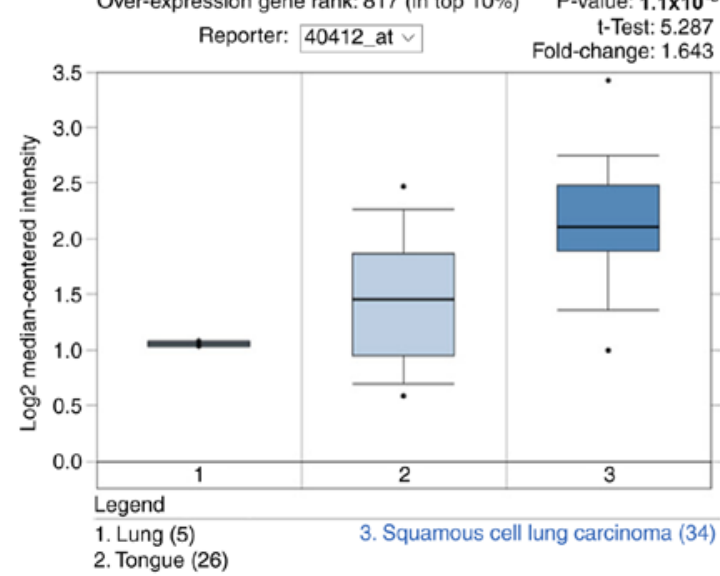

B Hou lung statistics

Over-expression gene rank: 27 (in top $1 \%$ ) P-value: $1.4 \times 10^{-27}$

Reporter: 203554_x_at $\mathrm{v}$ t-Test: 18.560

Fold-change: 5.665

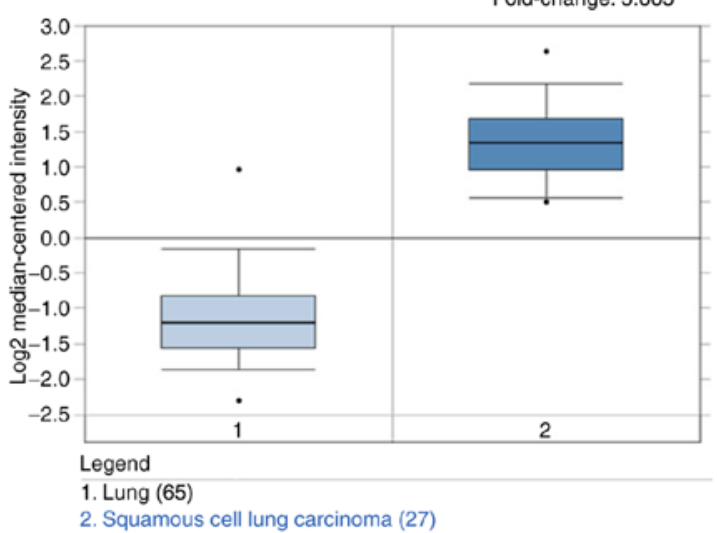

D Wachi lung statistics

Over-expression gene rank: 53 (in top $1 \%$ ) P-value: $2.0 \times 10^{-5}$ Reporter: $203554 \_x$ at $\square$ Fold-change: 3.275

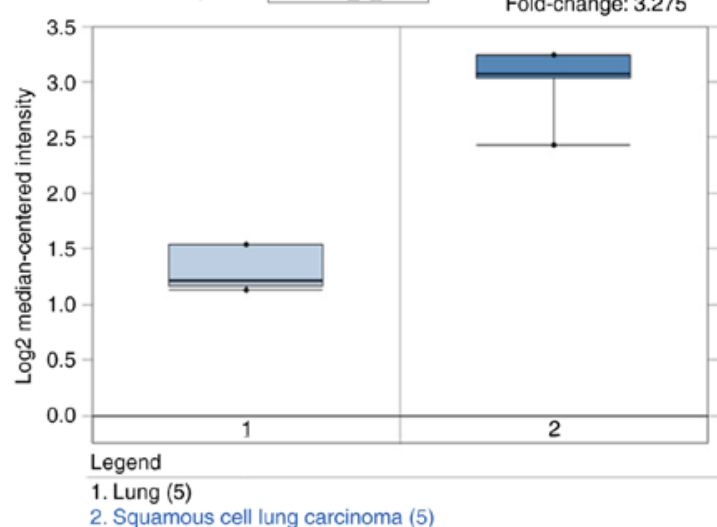

F Median rank p-Value Gene 53.0 2.00E-5 PTTG1

\begin{tabular}{|l|l|l|l|l|}
\hline 1 & 2 & 3 & 4 & 5 \\
\hline
\end{tabular}

Legend

1. Squamous cell lung carcinoma 4. Squamous cell lung carcinoma vs. normal Bhattacharjee lung, $\quad$ vs. normal Talbot lung, Cancer Proc Natl Acad Sci USA, 2001 Res, 2005

2. Squamous cell lung carcinoma vs. normal Garber lung, Proc Natl Acad Sci USA, $2001 \quad 5$. Squamous cell lung carcinoma 3. Squamous cell lung carcinoma vs. normal Wachi lung, vs. normal Hou lung. PLoS One, 2010

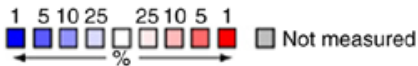

The rank for a gene is the median rank for that gene across each of the analyses. The p-Value for a gene is its p-Value for the median-ranked analysis.

Figure 3. Meta-analyses of PTTG1 expression in patients with LUSC. PTTG1 expression in patients with LUSC according to different datasets from the Oncomine database: (A) Bhattacharjee lung dataset; (B) Hou lung dataset; (C) Garber lung dataset; (D) Wachi lung dataset and (E) Talbot lung dataset. (F) The details of the meta-analyses of PTTG1 in patients with LUSC. The legends below the meta-analytical results contain detailed information on the selected datasets. PTTG, pituitary tumor transforming gene; LUSC, lung squamous cell carcinoma.

with a worse prognosis for all patients with NSCLC (HR, 1.21; 95\% CI, 1.07-1.37; log-rank $\mathrm{P}=2.9 \times 10^{-2}$; Fig. 5A). For patients with LUAD (HR, 1.16; 95\% CI, 0.92-1.46; log-rank P=2.0x 10-1) and patients with LUSC (HR, 1.06; 95\% CI, 0.83-1.33; log-rank $\left.\mathrm{P}=7.0 \times 10^{-1}\right)$, PTTG2 expression was not significantly associated with the prognosis of patients (Fig. 5B and C).
The associations between PTTG3P mRNA expression level and survival for all patients with NSCLC, patients with LUAD and patients with LUSC were evaluated. As presented in Fig. 6A and B, high expression of PTTG3P was significantly associated with unfavorable OS for all patients with NSCLC (HR, 1.57; 95\% CI, 1.38-1.78; log-rank $\mathrm{P}=2.9 \times 10^{-12}$ ) and 
Table I. Expression levels of PTTG1 in patients with non-small cell lung cancer from Oncomine database.

A, LUAD vs. normal

\begin{tabular}{|c|c|c|c|c|c|c|}
\hline Normal samples & Cancer samples & Reporter & Gene rank $(\%)$ & P-value & $\mathrm{t}$ & Fold change \\
\hline 17 & 132 & Bhattacharjee lung & 198 (top 3) & $2.0 \times 10^{-3}$ & 3.361 & 3.729 \\
\hline 19 & 20 & Stearman lung & 24 (top 1) & $4.4 \times 10^{-10}$ & 8.226 & 6.638 \\
\hline 65 & 45 & Hou lung & 154 (top 1) & $1.8 \times 10^{-14}$ & 9.338 & 2.984 \\
\hline 4 & 40 & Garber lung & 390 (top 4) & $2.0 \times 10^{-4}$ & 6.442 & 3.212 \\
\hline 49 & 58 & Landi lung & $65($ top 1$)$ & $3.6 \times 10^{-20}$ & 11.519 & 1.968 \\
\hline 30 & 27 & Su lung & 178 (top 2) & $1.4 \times 10^{-7}$ & 6.531 & 2.174 \\
\hline 20 & 226 & Okayama lung & 379 (top 2) & $1.2 \times 10^{-14}$ & 10.845 & 2.460 \\
\hline 58 & 58 & Selamat lung & 629 (top 4) & $3.5 \times 10^{-14}$ & 9.096 & 2.492 \\
\hline
\end{tabular}

B, LUSC vs. normal

\begin{tabular}{cclcrrr}
\hline Normal samples & Cancer samples & Reporter & Gene rank (\%) & P-value & $\mathrm{t}$ & Fold change \\
\hline 17 & 21 & Bhattacharjee lung & 46 (top 1) & $3.5 \times 10^{-8}$ & 7.028 \\
65 & 27 & Hou lung & 27 (top 1) & $1.4 \times 10^{-27}$ & 18.560 & 5.665 \\
4 & 13 & Garber lung & 241 (top 3) & $4.7 \times 10^{-5}$ & 8.912 & 4.863 \\
5 & 5 & Wachi lung & 53 (top 1) & $2.0 \times 10^{-5}$ & 10.763 \\
28 & 34 & Talbot lung & 817 (top 10) & $1.1 \times 10^{-6}$ & 5.287 & 1.643 \\
\hline
\end{tabular}

PTTG, pituitary tumor transforming gene; LUAD, lung adenocarcinoma; LUSC, lung squamous cell carcinoma.

patients with LUAD (HR, 1.81; 95\% CI, 1.43-2.30; log-rank $\left.\mathrm{P}=7.1 \times 10^{-7}\right)$. However, for patients with LUSC, PTTG3P was not significantly associated with OS (HR, 1.19; 95\% CI, 0.94-1.50; log-rank $\mathrm{P}=1.6 \times 10^{-1}$; Fig. $6 \mathrm{C}$ ). Taken together, these results indicated that the three PTTG family genes may be promising biomarkers that predict a poor prognosis in all patients with NSCLC. Additionally, PTTG1 and PTTG3P may also be two prospective prognostic biomarkers for patients with LUAD.

Associations between the prognostic values of PTTG family $m R N A$ expression and clinical stage. The associations between the prognostic values of the PTTG family mRNA expression levels and the clinical stage of patients with NSCLC were examined. Patients with clinical stage I NSCLC with high expression of PTTG1 (HR, 3.13; 95\% CI, 2.32-4.21; log-rank $\mathrm{P}=3.2 \times 10^{-15}$; Fig. 7A), PTTG2 (HR, 1.43; 95\% CI, 1.09-1.87; log-rank $\mathrm{P}=9.7 \times 10^{-3}$; Fig. 7B) and PTTG3P (HR, 2.73; 95\% CI, 2.04-3.65; log-rank $\mathrm{P}=2.3 \times 10^{-12}$; Fig. 7C) exhibited worse OS compared with patients with low PTTG1 expression. High expression of PTTG2 indicated a poor prognosis in patients with clinical stage II NSCLC compared with low PTTG2 expression (HR, 1.61; 95\% CI, 1.12-2.33; log-rank $\mathrm{P}=1.0 \times 10^{-2}$; Fig. 7E). PTTG1 (HR, 1.26; 95\% CI, 0.87-1.82; log-rank $\mathrm{P}=2.2 \times 10^{-1}$; Fig. 7D) and PTTG3P (HR, 1.18; 95\% CI, 0.82-1.70; log-rank $\mathrm{P}=3.8 \times 10^{-1}$; Fig. 7F) did not demonstrate any significant effects on the OS of patients with stage II NSCLC. For patients with clinical stage III NSCLC, PTTG1 (HR, 0.89; 95\% CI, 0.51-1.53; log-rank P=6.6×10-1; Fig. 7G), PTTG2 (HR, 0.74; 95\% CI, 0.42-1.27; log-rank P=2.7x10 $0^{-1}$; Fig. 7H) and PTTG3P (HR, 1.29; 95\% CI, 0.75-2.22; log-rank
$\mathrm{P}=3.6 \times 10^{-1}$; Fig. 7I) expression levels exhibited no significant associations with prognosis. These results suggested that PTTG family genes may be effective prognostic biomarkers for patients with clinical stage I NSCLC.

Associations between the prognostic values of PTTG family $m R N A$ expression and chemotherapy or radiotherapy. Chemotherapy and radiotherapy are two major therapeutic strategies for treating different cancer types, including NSCLC, particularly for patients with advanced stage disease. The present study further investigated the associations between the prognostic roles of the mRNA expression levels of PTTG family genes and chemotherapy and radiotherapy in NSCLC. High expression levels of PTTG1 (HR, 1.58; 95\% CI, 1.13-2.22; log-rank $\mathrm{P}=7.0 \times 10^{-3}$ ) and PTTG3P (HR, 1.45; 95\% CI, 1.03-2.03; log-rank $\mathrm{P}=3.0 \times 10^{-2}$ ) were significantly associated with OS of patients with NSCLC without chemotherapy (Table II). However, none of the PTTG family gene expression levels were significantly associated with OS of patients with or without radiotherapy (Table III).

Associations between the prognostic values of PTTG family $m R N A$ expression levels and other clinicopathological features. The associations of individual PTTG family genes with other clinicopathological features, including pathological grade (Fig. 8), lymph node status (Table IV), smoking status (Table V) and sex (Table VI), were determined. Fig. 8 presents the prognostic values of PTTG family genes in NSCLC based on various pathological grades; none of the genes demonstrated a significant association with OS of patients with grade I, II 
Table II. Correlation of PTTG family with chemotherapy of patients with non-small cell lung cancer.

\begin{tabular}{|c|c|c|c|c|c|c|c|}
\hline $\begin{array}{l}\text { PTTG } \\
\text { family member }\end{array}$ & Affymetrix ID & Chemotherapy & $\begin{array}{c}\text { Low } \\
\text { expression }(\mathrm{N})\end{array}$ & $\begin{array}{c}\text { High } \\
\text { expression }(\mathrm{N})\end{array}$ & HR & $95 \% \mathrm{CI}$ & $\begin{array}{c}\text { Log-rank } \\
\text { P-value }\end{array}$ \\
\hline \multirow[t]{2}{*}{ PTTG1 } & 203554_x_at & No & 155 & 155 & 1.58 & $1.13-2.22$ & $7.0 \times 10^{-3 a}$ \\
\hline & 203554_x_at & Yes & 88 & 88 & 0.95 & $0.63-1.45$ & $8.3 \times 10^{-1}$ \\
\hline \multirow[t]{2}{*}{ PTTG2 } & 214557_at & No & 158 & 152 & 1.16 & $0.83-1.63$ & $3.7 \times 10^{-1}$ \\
\hline & 214557_at & Yes & 88 & 88 & 0.93 & $0.62-1.4$ & $7.3 \times 10^{-1}$ \\
\hline \multirow[t]{2}{*}{ PTTG3P } & 208511_at & No & 156 & 154 & 1.45 & $1.03-2.03$ & $3.0 \times 10^{-2 \mathrm{a}}$ \\
\hline & 208511_at & Yes & 89 & 87 & 0.81 & $0.54-1.22$ & $3.1 \times 10^{-1}$ \\
\hline
\end{tabular}

${ }^{\text {a }}<<0.05$. PTTG, pituitary tumor transforming gene.

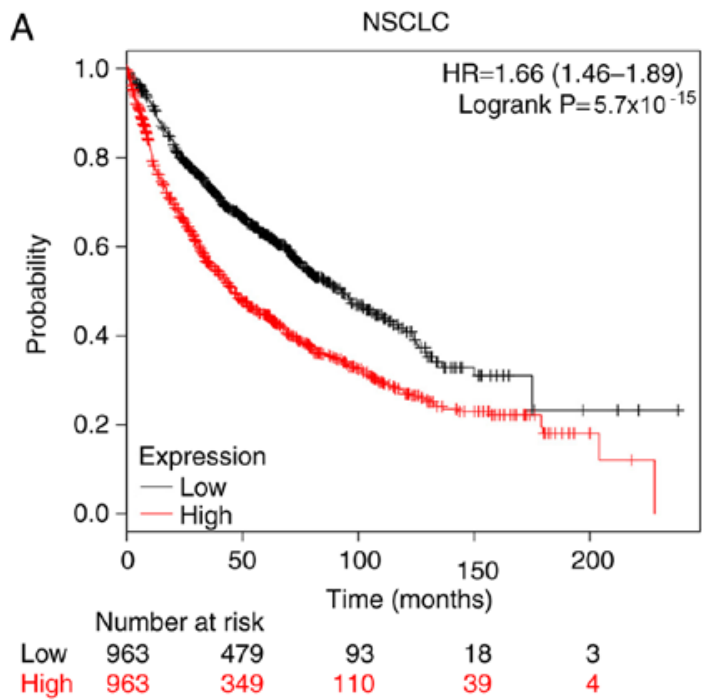

B

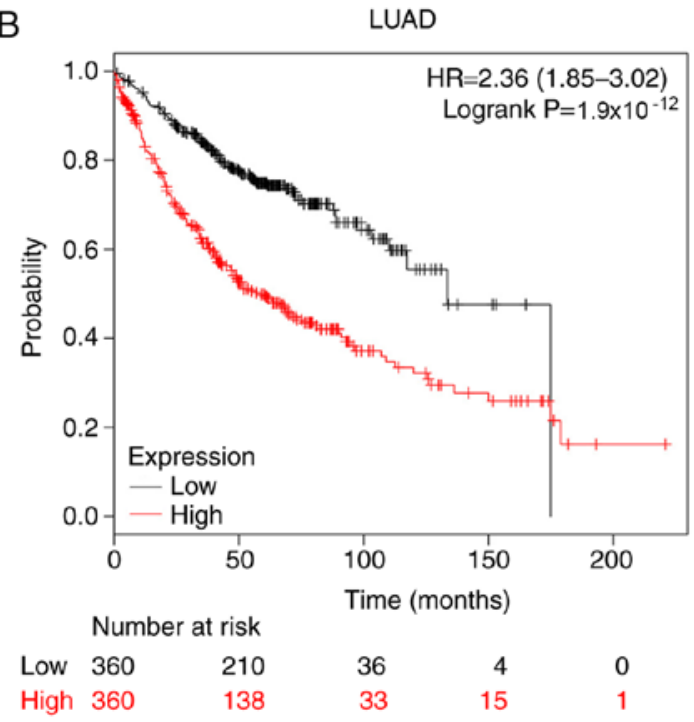

C

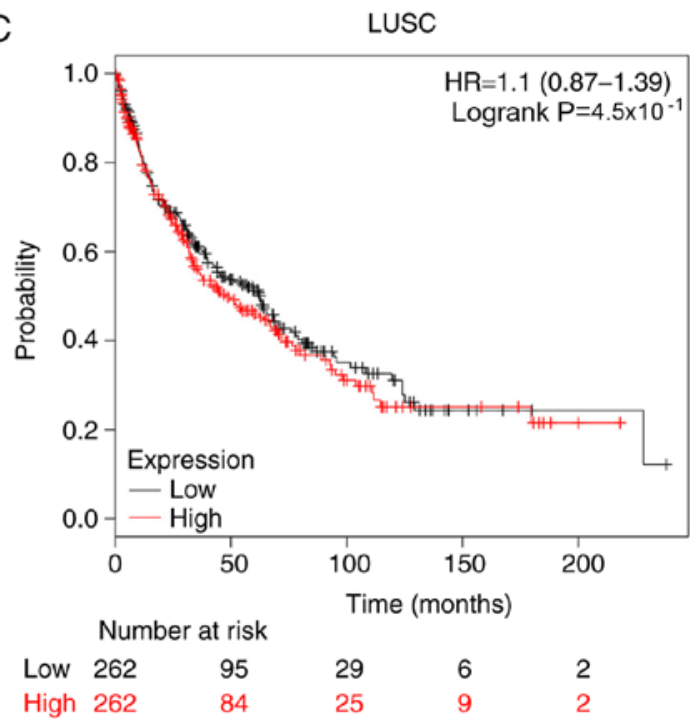

Figure 4. Prognostic values of PTTG1 mRNA expression in all patients with NSCLC, patients with LUAD and patients with LUSC. (A-C) The prognostic value of PTTG1 mRNA expression in (A) all patients with NSCLC, (B) patients with LUAD and (C) patients with LUSC. PTTG, pituitary tumor transforming gene; NSCLC, non-small cell lung cancer; LUAD, lung adenocarcinoma; LUSC, lung squamous cell carcinoma.

or III NSCLC, which may have occurred partially due to the relatively limited sample size. The data presented in Table IV demonstrated the associations between the prognostic values of
PTTG family mRNA expression levels and lymph node status of patients with NSCLC. A high expression of PTTG1 (HR, 1.39; 95\% CI, 1.12-1.71; log-rank $\mathrm{P}=2.3 \times 10^{-3}$ ) was significantly 
Table III. Correlation of PTTG family with radiotherapy of patients with non-small cell lung cancer.

\begin{tabular}{lccccccc}
\hline $\begin{array}{l}\text { PTTG } \\
\text { family member }\end{array}$ & Affymetrix ID & Radiotherapy & $\begin{array}{c}\text { Low } \\
\text { expression (N) }\end{array}$ & $\begin{array}{c}\text { High } \\
\text { expression (N) }\end{array}$ & HR & $\begin{array}{c}\text { S5\% CI } \\
\text { P-value }\end{array}$ \\
\hline PTTG1 & 203554_x_at & No & 137 & 134 & 1.22 & $0.86-1.75$ & $2.6 \times 10^{-1}$ \\
& 203554_x_at & Yes & 35 & 35 & 0.95 & $0.55-1.63$ & $8.4 \times 10^{-1}$ \\
PTTG2 & 214557_at & No & 136 & 135 & 1.04 & $0.73-1.49$ & $8.2 \times 10^{-1}$ \\
& 214557_at & Yes & 36 & 34 & 0.87 & $0.51-1.49$ & $6.2 \times 10^{-1}$ \\
PTTG3P & 208511_at & No & 136 & 135 & 1.11 & $0.77-1.58$ & $5.8 \times 10^{-1}$ \\
& 208511_at & Yes & 35 & 35 & 1.15 & $0.68-1.96$ & $6.0 \times 10^{-1}$ \\
\hline
\end{tabular}

PTTG, pituitary tumor transforming gene.

A

NSCLC

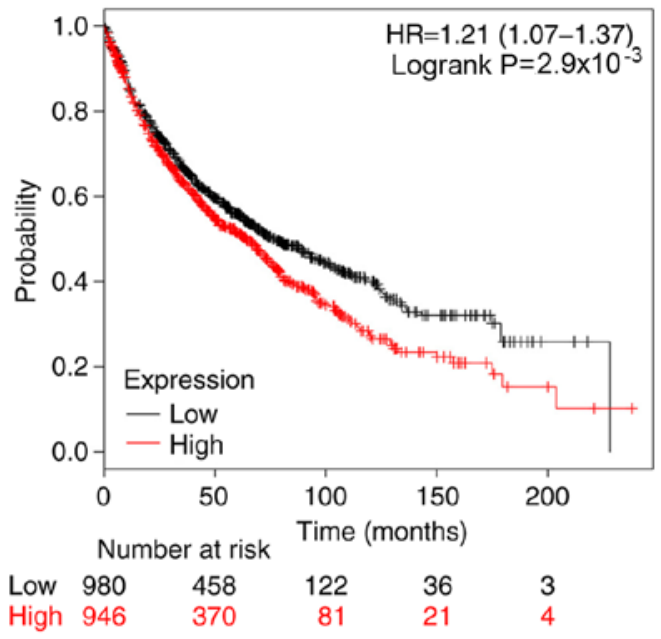

B

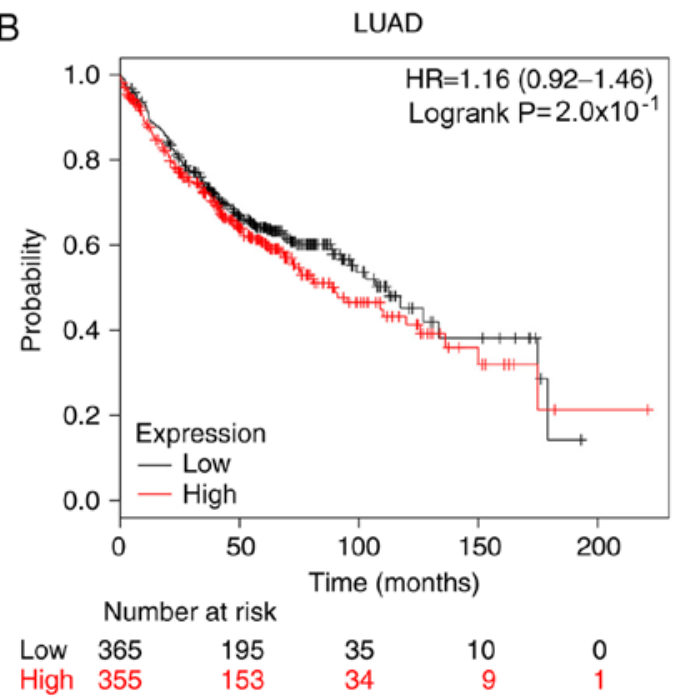

C

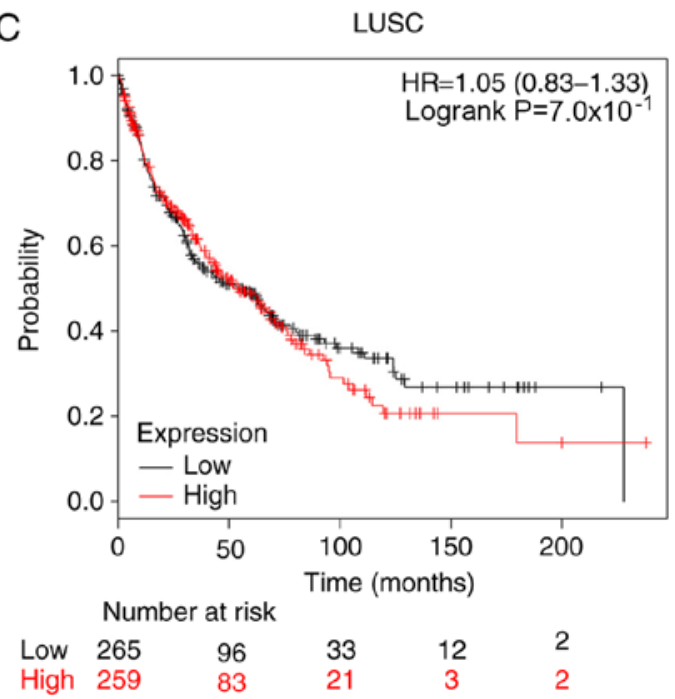

Figure 5. Prognostic values of PTTG2 mRNA expression in all patients with NSCLC, patients with LUAD and patients with LUSC. (A-C) The prognostic value of PTTG2 mRNA expression in (A) all patients with NSCLC, (B) patients with LUAD and (C) patients with LUSC. PTTG, pituitary tumor transforming gene; NSCLC, non-small cell lung cancer; LUAD, lung adenocarcinoma; LUSC, lung squamous cell carcinoma.

associated with poor OS for patients with NSCLC without invasive and/or metastatic lymph nodes (lymph node, 0 ). However, PTTG2 and PTTG3P were not associated with NSCLC lymph node status. Table V presents the associations of the PTTG family with the smoking history of patients with NSCLC. Compared with patients with NSCLC with low expression of PTTG1, high expression of PTTG1 indicated a worse prognosis in patients with NSCLC who had never smoked (HR, 3.03; 
Table IV. Correlation of PTTG family with lymph node status of patients with non-small cell lung cancer.

\begin{tabular}{lccccccc}
\hline $\begin{array}{l}\text { PTTG family } \\
\text { member }\end{array}$ & Affymetrix ID & $\begin{array}{c}\text { Lymph node } \\
\text { status }\end{array}$ & $\begin{array}{c}\text { Low } \\
\text { expression (N) }\end{array}$ & $\begin{array}{c}\text { High } \\
\text { expression (N) }\end{array}$ & HR & 95\% CI & $\begin{array}{c}\text { Log-rank } \\
\text { P-value }\end{array}$ \\
\hline PTTG1 & 203554_x_at & 0 & 390 & 391 & 1.39 & $1.12-1.71$ & $2.3 \times 10^{-3 a}$ \\
& 214775_at & 1 & 126 & 126 & 1.21 & $0.89-1.66$ & $2.3 \times 10^{-1}$ \\
& 208511_at & 2 & 56 & 55 & 1.01 & $0.67-1.51$ & $9.7 \times 10^{-1}$ \\
PTTG2 & 203554_x_at & 0 & 391 & 390 & 1.06 & $0.86-1.31$ & $5.7 \times 10^{-1}$ \\
& 214775_at & 1 & 129 & 123 & 1.22 & $0.89-1.67$ & $2.1 \times 10^{-1}$ \\
& 208511_at & 2 & 56 & 55 & 1.23 & $0.82-1.84$ & $3.2 \times 10^{-1}$ \\
PTTG3P & 203554_x_at & 0 & 391 & 390 & 1.12 & $0.9-1.38$ & $3.1 \times 10^{-1}$ \\
& 214775_at & 1 & 128 & 124 & 1.24 & $0.91-1.69$ & $1.8 \times 10^{-1}$ \\
& 208511_at & 2 & 56 & 55 & 1.32 & $0.88-1.97$ & $1.8 \times 10^{-1}$ \\
\hline
\end{tabular}

${ }^{\mathrm{a}} \mathrm{P}<0.05$. PTTG, pituitary tumor transforming gene.

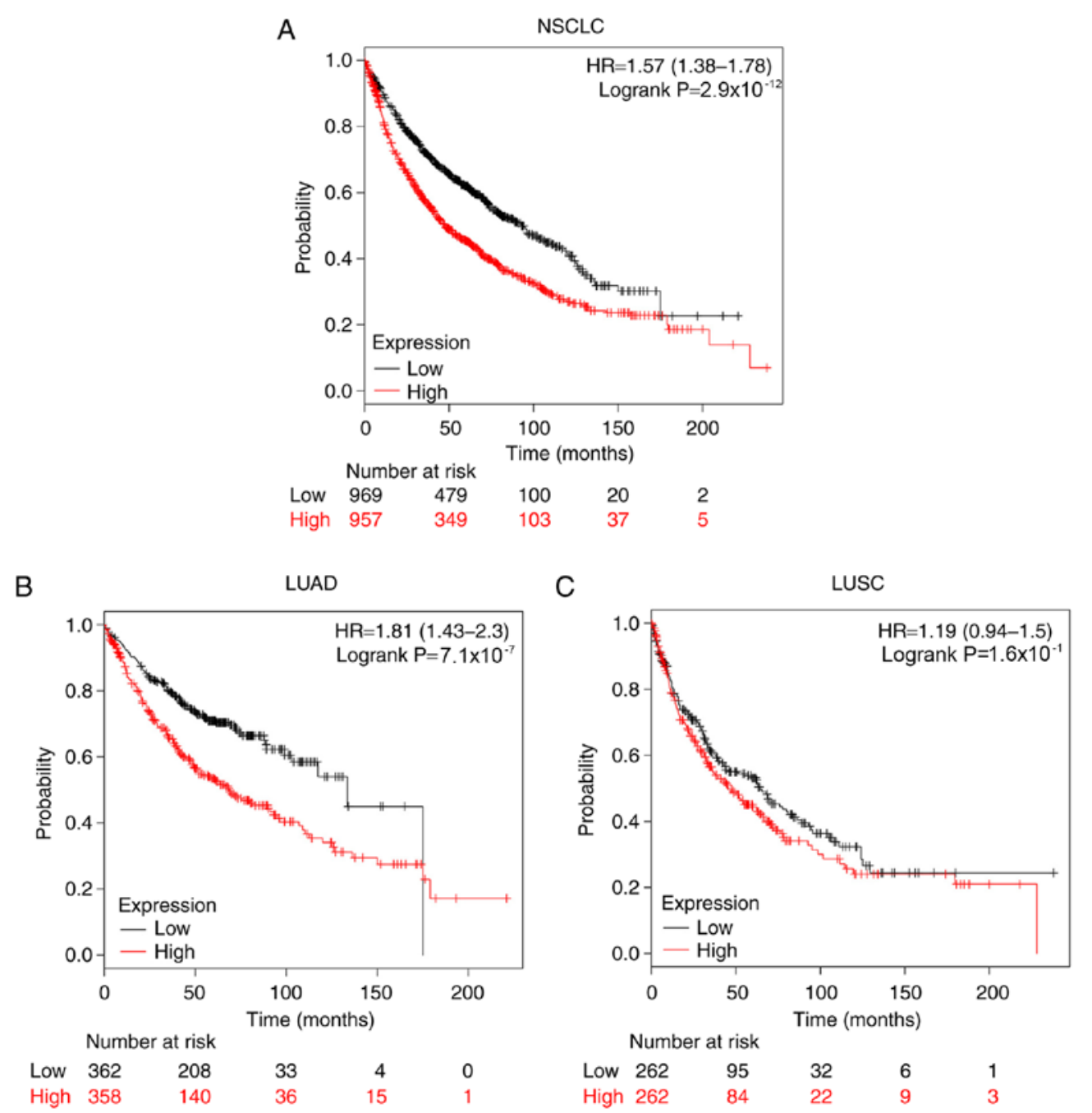

Figure 6. Prognostic values of PTTG3P mRNA expression in all patients with NSCLC, patients with LUAD and patients with LUSC. (A-C) The prognostic value of PTTG3P mRNA expression in (A) all patients with NSCLC, (B) patients with LUAD and (C) patients with LUSC. PTTG, pituitary tumor transforming gene; NSCLC, non-small cell lung cancer; LUAD, lung adenocarcinoma; LUSC, lung squamous cell carcinoma.

95\% CI, 1.63-5.62; log-rank $\left.\mathrm{P}=2.2 \times 10^{-4}\right)$ or smoked $(\mathrm{HR}, 1.32$; 95\% CI, 1.07-1.62; log-rank $\left.\mathrm{P}=8.9 \times 10^{-3}\right)$. Patients with NSCLC with a high expression of PTTG2 who had smoked (HR, 1.47;
95\% CI, 1.19-1.81; log-rank $\mathrm{P}=2.8 \times 10^{-4}$ ) and never-smoked (HR, 2.10; 95\% CI, 1.18-3.75; log-rank $\mathrm{P}=1.0 \times 10^{-2}$ ) exhibited a shorter OS time compared with patients with NSCLC with low 
A
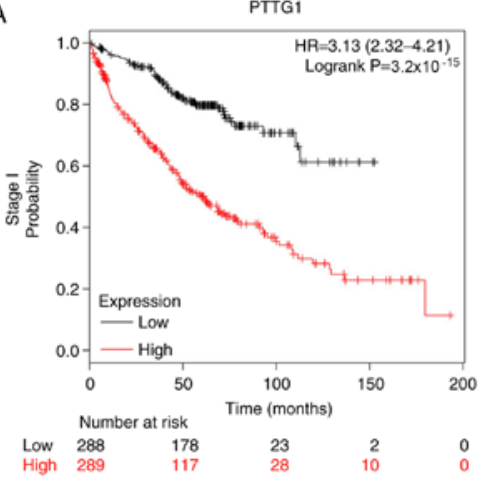

C
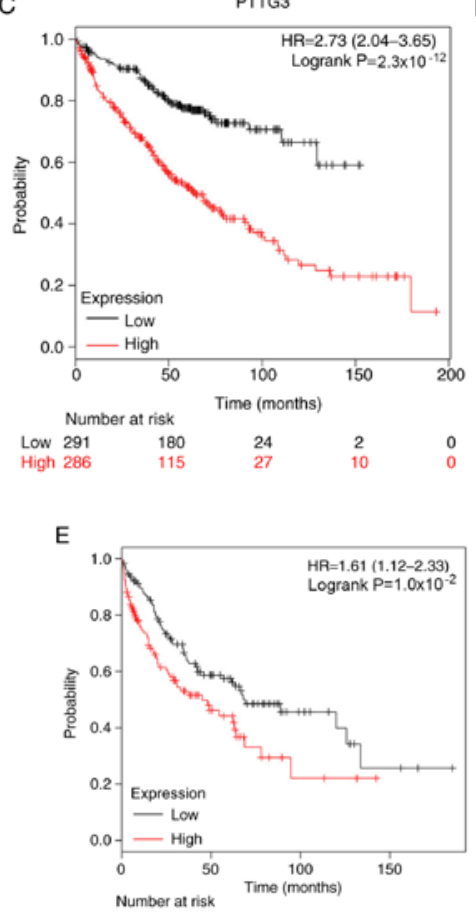

$\begin{array}{lllll}\text { Low } & 127 & 52 & 11 & 3 \\ \text { High } & 117 & 24 & 3 & 0\end{array}$

G

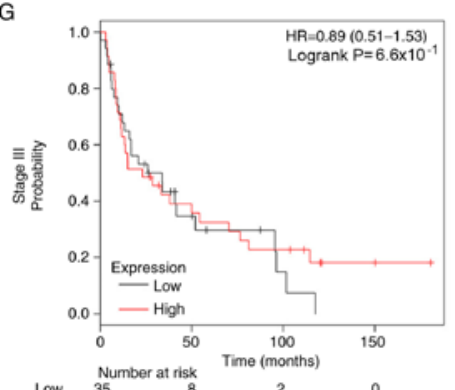

$\begin{array}{lrrr} & \text { Number at risk } & \text { Time (mmon } \\ \text { Low } & 35 & 8 & \\ \text { High } & 35 & 11 & \end{array}$
BTTG2

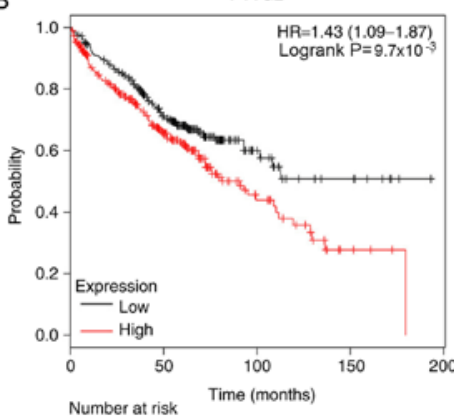

$\begin{array}{lllll}\text { Low } 288 & 170 & 26 & 8 & 0 \\ \text { High } 289 & 125 & 25 & 4 & 0\end{array}$

D

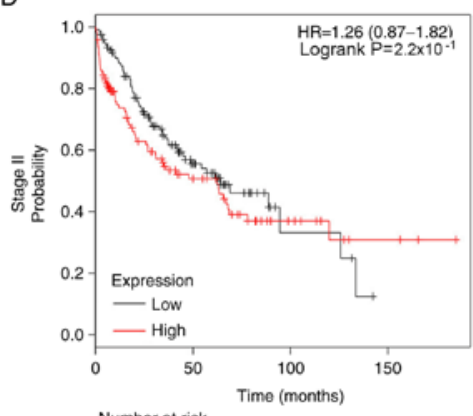

\begin{tabular}{lrrrr}
\multicolumn{5}{c}{ Number at risk } \\
Low & 122 & 40 & 4 & 0 \\
High & 122 & 36 & 10 & 3
\end{tabular}

$\mathrm{F}$

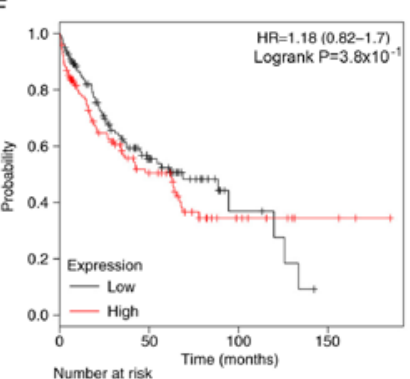

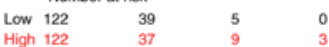

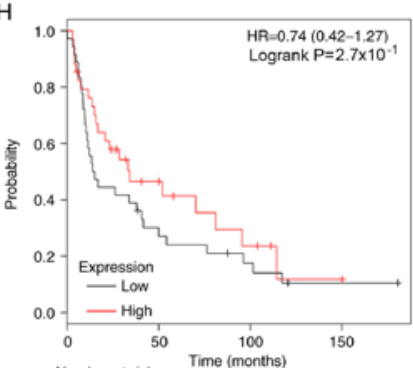

$\begin{array}{llccc} & \text { Number at risk } & \text { Time (months) } \\ \text { Low } & 36 & 9 & 5 \\ \text { High } & 34 & 10 & 4\end{array}$

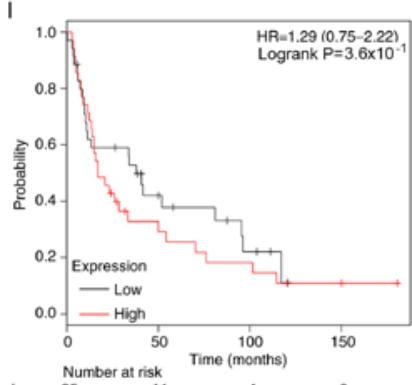

\begin{tabular}{rrrrr} 
& \multicolumn{2}{c}{ Number at risk } & Time (months) \\
Low & 35 & 11 & 4 \\
High & 35 & 8 & 5
\end{tabular}

Figure 7. Prognostic values of the mRNA expression PTTG family in NSCLC patients based on different clinical stages. (A-C) The prognostic value of (A) PTTG1, (B) PTTG2 and (C) PTTG3P mRNA expression in patients with clinical stage I NSCLC. (D-F) The prognostic value of (D) PTTG1, (E) PTTG2 and (F) PTTG3P mRNA expression in patients with clinical stage II NSCLC. (G-I) The prognostic value of (G) PTTG1, (H) PTTG2 and (I) PTTG3P mRNA expression in patients with clinical stage III NSCLC. PTTG, pituitary tumor transforming gene; NSCLC, non-small cell lung cancer. 


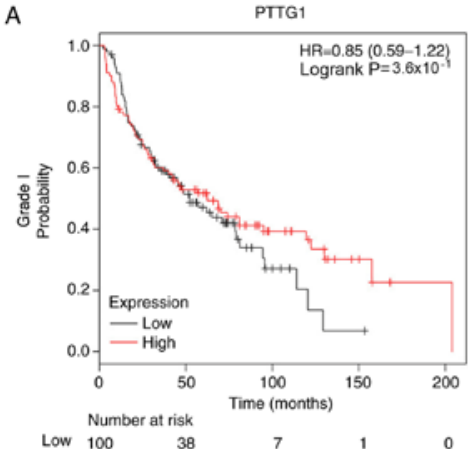

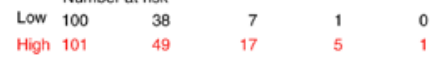

C
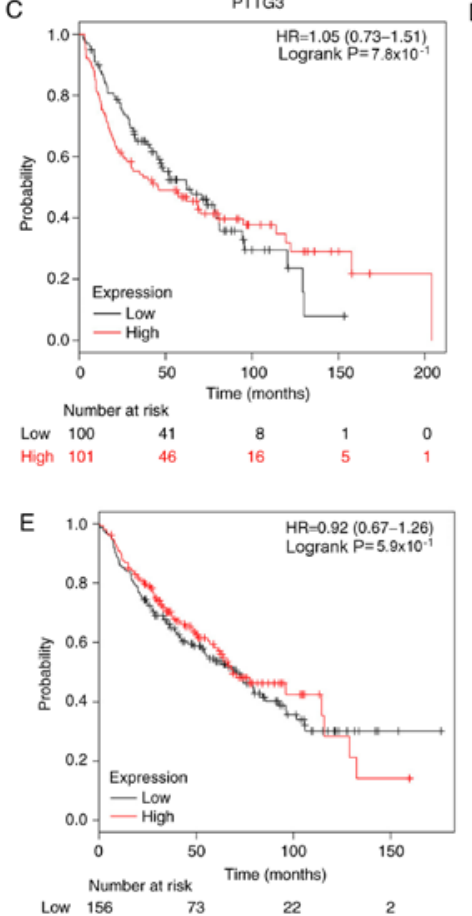

B

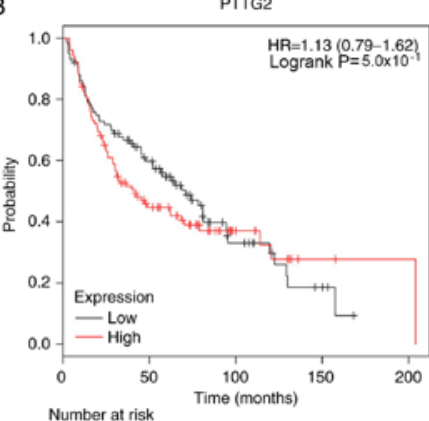

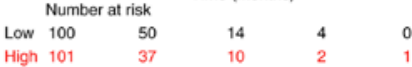

D

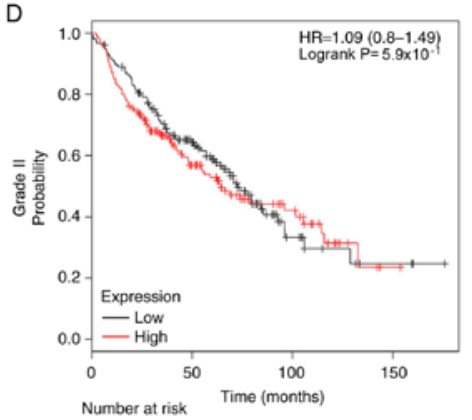

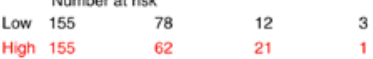

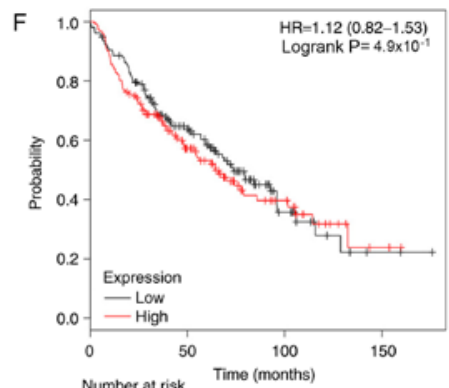

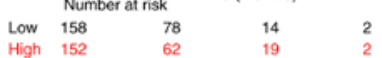

G

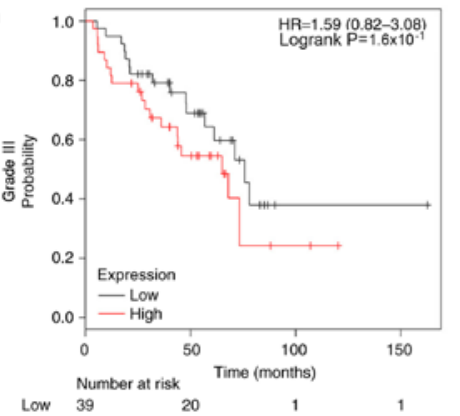

$\mathrm{H} \quad 1.0 \mathrm{H}$
$\begin{gathered}\mathrm{HR}=0.82(0.43-1.6) \\ \text { Logrank } P=5.7 \times 10^{-1}\end{gathered}$

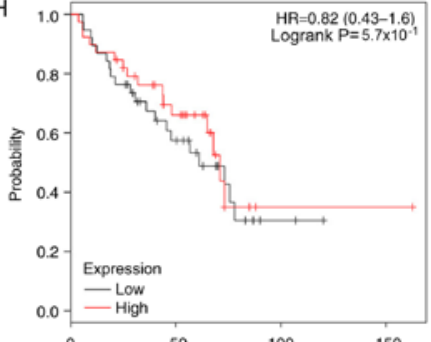

$\begin{array}{llll}50 & 100 & 150\end{array}$

Number at risk Time (months)

$\begin{array}{lll}\text { Low } & 38 & 17 \\ \text { High } & 39 & 19\end{array}$

$\mathrm{HR}=1.43(0.73-2.78)$

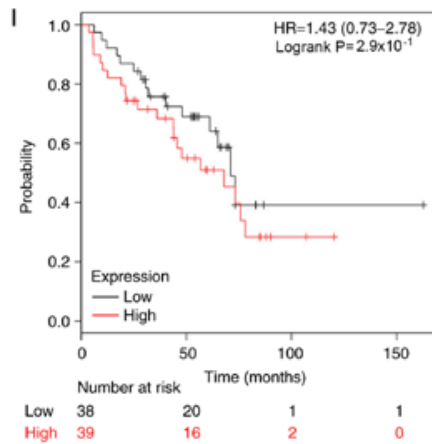

Figure 8. The prognostic values of the mRNA expression PTTG family in NSCLC patients based on different pathological grades. (A-C) The prognostic value of (A) PTTG1, (B) PTTG2 and (C) PTTG3P mRNA expression in patients with pathological grade I NSCLC. (D-F) The prognostic value of (D) PTTG1, (E) PTTG2 and (F) PTTG3P mRNA expression in patients with pathological grade II NSCLC. (G-I) The prognostic value of (G) PTTG1, (H) PTTG2 and (I) PTTG3P mRNA expression in patients with pathological grade III NSCLC. PTTG, pituitary tumor transforming gene; NSCLC, non-small cell lung cancer. 
Table V. Correlation of PTTG family with smoking history of patients with non-small cell lung cancer.

\begin{tabular}{lclccccc}
\hline $\begin{array}{l}\text { PTTG } \\
\text { family member }\end{array}$ & Affymetrix ID & Smoking status & $\begin{array}{c}\text { Low } \\
\text { expression (N) }\end{array}$ & $\begin{array}{c}\text { High } \\
\text { expression (N) }\end{array}$ & HR & $\begin{array}{c}\text { Log-rank } \\
\text { P-value }\end{array}$ \\
\hline PTTG1 & 203554_x_at & Never smoked & 102 & 103 & 3.03 & $1.63-5.62$ & $2.2 \times 10^{-4 a}$ \\
& 203554_x_at & Smoked & 410 & 410 & 1.32 & $1.07-1.62$ & $8.9 \times 10^{-3 a}$ \\
PTTG2 & 214557_at & Never smoked & 102 & 103 & 2.1 & $1.18-3.75$ & $1.0 \times 10^{-2 a}$ \\
& 214557_at & Smoked & 423 & 397 & 1.47 & $1.19-1.81$ & $2.8 \times 10^{-4 a}$ \\
PTTG3P & 208511_at & Never smoked & 105 & 100 & 3.11 & $1.70-5.71$ & $1.1 \times 10^{-4 a}$ \\
& 208511_at & Smoked & 413 & 407 & 1.41 & $1.15-1.74$ & $1.1 \times 10^{-3 a}$ \\
\hline
\end{tabular}

${ }^{\text {a }}<0$.05.PTTG, pituitary tumor transforming gene.

Table VI. Correlation of PTTG family with the sex of non-small cell lung cancer patients.

\begin{tabular}{lclccccc}
\hline $\begin{array}{l}\text { PTTG family } \\
\text { member }\end{array}$ & Affymetrix ID & Sex & $\begin{array}{c}\text { Low } \\
\text { expression (N) }\end{array}$ & $\begin{array}{c}\text { High } \\
\text { expression (N) }\end{array}$ & HR & $\begin{array}{c}\text { Log-rank } \\
\text { P-value }\end{array}$ \\
\hline PTTG1 & 203554_x_at & Female & 359 & 356 & 1.87 & $1.47-2.38$ & $1.7 \times 10^{-7 \mathrm{a}}$ \\
& 203554_x_at & Male & 550 & 550 & 1.53 & $1.31-1.79$ & $1.2 \times 10^{-7 \mathrm{a}}$ \\
PTTG2 & 214557_at & Female & 364 & 351 & 1.34 & $1.06-1.69$ & $1.4 \times 10^{-2 a}$ \\
& 214557_at & Male & 580 & 520 & 1.24 & $1.06-1.46$ & $6.9 \times 10^{-3 \mathrm{a}}$ \\
PTTG3P & 208511_at & Female & 359 & 356 & 1.81 & $1.43-2.29$ & $6.6 \times 10^{-7 \mathrm{a}}$ \\
& 208511_at & Male & 556 & 544 & 1.45 & $1.24-1.70$ & $4.2 \times 10^{-6 a}$ \\
\hline
\end{tabular}

${ }^{\mathrm{a}} \mathrm{P}<0.05$. PTTG, pituitary tumor transforming gene.

expression of PTTG2. Additionally, high expression of PTTG3P was also significantly associated with the OS or patients who had never-smoked (HR, 3.11; 95\% CI, 1.70-5.71; log-rank $\mathrm{P}=1.1 \times 10^{-4}$ ) and smoked (HR, 1.41; 95\% CI, 1.15-1.74; log-rank $\left.\mathrm{P}=1.1 \times 10^{-3}\right)$. High expression of PTTG1, PTTG2 and PTTG3P was significantly associated with OS of both female and male patients with NSCLC (PTTG1-female: HR, 1.87; 95\% CI, 1.47-2.38; log-rank $\mathrm{P}=1.7 \times 10^{-7}$; PTTG1-male: HR, 1.53; 95\% CI, 1.31-1.79; log-rank $\mathrm{P}=1.2 \times 10^{-7}$; PTTG2-female: HR, 1.34; 95\% CI, 1.06-1.69; log-rank $\mathrm{P}=1.4 \times 10^{-2}$; PTTG2-male: HR, 1.24; 95\% CI, 1.06-1.46; log-rank $\mathrm{P}=6.9 \times 10^{-3}$; PTTG3P-female: HR, 1.81; 95\% CI, 1.43-2.29; log-rank $\mathrm{P}=6.6 \times 10^{-7}$; PTTG3P-male: $\mathrm{HR}, 1.45 ; 95 \% \mathrm{CI}, 1.24-1.70$; log-rank $\mathrm{P}=4.2 \times 01^{-6}$; Table VI).

\section{Discussion}

Lung cancer is the leading cause of cancer-associated mortality worldwide, which is associated with significant health and financial burdens (1). As the most common type or lung cancer, rapid improvements in the diagnosis, treatment and prognosis of NSCLC is important. The PTTG family, which comprises PTTG1, PTTG2 and PTTG3P, is a newly identified gene class. Among the three homologous genes, PTTG1 has been the most extensively studied and has been identified to be closely associated with the onset and progression of multiple human cancer types, including pituitary tumor (22), malignant glioma (7), thyroid (23), breast (24), ovarian (25), bladder (8), prostate (9) and lung cancer (26-30).
Honda et al (30) have demonstrated that PTTG1 is significantly upregulated in NSCLC and its overexpression serves a role in the genesis and progression of NSCLC. However, to the best or our knowledge, a systematic analysis regarding the expression and prognostic role of PTTG1 in NSCLC has not been previously performed. In addition, PTTG2 and PTTG3P have been reported to be associated with tumor development $(12,13)$; to the best of our knowledge, no previous study has investigated their expression and roles in NSCLC. Therefore, the present study systematically investigated the expression and prognostic roles of the PTTG family genes in NSCLC.

A comprehensive analysis of the mRNA expression of the PTTG family genes in NSCLC was performed in the present study using the GEPIA, UALCAN and Oncomine databases. The results demonstrated that PTTG1 was significantly upregulated in cancer tissue compared with normal tissue, which was in accordance with the results of previous studies on other types of cancer (7-9). By contrast, for PTTG2 and PTTG3P expression, the data from the three databases were inconsistent. The results of the GEPIA and Oncomine database analysis suggested that there were no significant differences in PTTG2 and PTTG3P expression between NSCLC and normal lung tissues. However, the results from the UALCAN database indicated an upregulation of PTTG2 and PTTG3P in NSCLC compared with normal tissue. Therefore, further studies on the expression of these genes in NSCLC are required to confirm these results. 
The Kaplan Meier-plotter database was used to perform a broad assessment of the prognostic roles of the PTTG family genes in patients with NSCLC. The results demonstrated that patients with NSCLC (1,924 samples) with high PTTG1, PTTG2 and PTTG3P expression exhibited a shorter OS time compared with healthy controls. In different subtypes of patients with NSCLC, PTTG1 and PTTG3P may serve as promising prognostic biomarkers, as their mRNA expression levels were significantly associated with the prognosis of patients with LUAD. However, for patients with LUAD and LUSC, PTTG2 was not significantly associated with prognosis. Only 720 and 524 clinical samples were included in the analysis of the prognostic roles of PTTG2 in patients with LUAD and LUSC, respectively; the relatively small sample counts may have influenced the results.

The present study further determined the associations of the prognostic values of the mRNA expression of PTTG family genes with clinicopathological features, including clinical stage, pathological grade, lymph node metastasis, smoking history and sex. The associations between the levels of PTTG family mRNA expression and chemotherapy or radiotherapy were also assessed. The findings demonstrated that a high expression of PTTG family genes indicated a poor prognosis in patients with clinical stage I disease, patients who had smoked, patients who had never-smoked. No differences were observed in terms of the sex of the patients in the prognosis of patients with NSCLC with high expression of PTTG family genes. High expression levels of PTTG1 and PTTG3P were associated with short survival time of patients with NSCLC who had not received chemotherapy. PTTG family genes demonstrated no significant association with radiotherapy, pathological grade and lymph node status, partially due to the relatively limited sample size. These results may inform the selection of therapeutic choices for NSCLC patients with various PTTG family gene expression levels. Future studies with a larger sample size are required to further reveal the associations of the expression levels of PTTG family genes with these clinicopathological features in patients with NSCLC. Of note, in addition to LUAD and LUSC subtypes, NSCLC also has other subtypes, such as lung adenosquamous carcinoma and large cell lung cancer. Therefore, although PTTG family exhibited unfavorable prognostic values in all NSCLC patients, they may have various prognostic values in LUAD or LUSC.

In conclusion, the results of the present study suggest that PTTG family genes, particularly PTTG1, are significantly overexpressed in LUAD and LUSC. In addition, increased expression of PTTG family genes may serve as promising prognostic biomarkers for patients with NSCLC.

\section{Acknowledgements}

Not applicable.

\section{Funding}

This study was supported by the 2017 Science and Technology Innovation Team Project of Zhengzhou Railway Vocational and Technical College (grant no. 17060001).

\section{Availability of data and materials}

All data generated or analyzed during this study are included in this published article.

\section{Authors' contributions}

WL and JC conceived and designed the study. SY, XW and JL wrote the manuscript. SY, XW and JL performed gene expression analysis, survival analysis and prepared figures and tables. $\mathrm{BD}$ and $\mathrm{KS}$ interpreted the results. $\mathrm{BD}, \mathrm{KS}$ and $\mathrm{WL}$ revised the manuscript. All authors read and approved the final manuscript.

\section{Ethics approval and consent to participate}

Not applicable.

\section{Patient consent for publication}

Not applicable.

\section{Competing interests}

The authors declare that they have no competing interests.

\section{References}

1. Chen W, Zheng R, Baade PD, Zhang S, Zeng H, Bray F, Jemal A, $\mathrm{Yu}$ XQ and He J: Cancer statistics in China, 2015. CA Cancer J Clin 66: 115-132, 2016.

2. Liu K, Jin M, Xiao L, Liu H and Wei S: Distinct prognostic values of mRNA expression of glutathione peroxidases in non-small cell lung cancer. Cancer Manag Res 10: 2997-3005, 2018.

3. Jones CM, Brunelli A, Callister ME and Franks KN: Multimodality treatment of advanced non-small cell lung cancer: Where are we with the evidence? Curr Surg Rep 6: 5, 2018.

4. You Q, Guo H and Xu D: Distinct prognostic values and potential drug targets of ALDH1 isoenzymes in non-small-cell lung cancer. Drug Des Devel Ther 9: 5087-5097, 2015.

5. Chen L, Puri R, Lefkowitz EJ and Kakar SS: Identification of the human pituitary tumor transforming gene (hPTTG) family: Molecular structure, expression, and chromosomal localization. Gene 248: 41-50, 2000.

6. Vlotides G, Eigler T and Melmed S: Pituitary tumor-transforming gene: Physiology and implications for tumorigenesis. Endocr Rev 28: 165-186, 2007.

7. Su X, Chen J, Ni L, Shi W, Shi J, Liu X, Zhang Y, Gong P, Zhu H and Huang Q: Inhibition of PTTG1 expression by microRNA suppresses proliferation and induces apoptosis of malignant glioma cells. Oncol Lett 12: 3463-3471, 2016.

8. Xiang W, Wu X, Huang C, Wang M, Zhao X, Luo G, Li Y, Jiang G, Xiao X and Zeng F: PTTG1 regulated by miR-146a-3p promotes bladder cancer migration, invasion, metastasis and growth. Oncotarget 8: 664-678, 2017.

9. Zhang Z, Jin B, Jin Y, Huang S, Niu X, Mao Z and Xin D: PTTG1, A novel androgen responsive gene is required for androgen-induced prostate cancer cell growth and invasion. Exp Cell Res 350: 1-8, 2017.

10. Li H, Yin C, Zhang B, Sun Y, Shi L, Liu N, Liang S, Lu S, Liu Y, Zhang J, et al: PTTG1 promotes migration and invasion of human non-small cell lung cancer cells and is modulated by miR-186. Carcinogenesis 34: 2145-2155, 2013.

11. Li WH, Chang L, Xia YX, Wang L, Liu YY, Wang YH, Jiang Z, Xiao J and Wang ZR: Knockdown of PTTG1 inhibits the growth and invasion of lung adenocarcinoma cells through regulation of TGFB1/SMAD3 signaling. Int J Immunopathol Pharmacol 28: 45-52, 2015.

12. Guo Y, Shao Y, Chen J, Xu S, Zhang X and Liu H: Expression of pituitary tumor-transforming 2 in human glioblastoma cell lines and its role in glioblastoma tumorigenesis. Exp Ther Med 11: 1847-1852, 2016. 
13. Weng W, Ni S, Wang Y, Xu M, Zhang Q, Yang Y, Wu Y, Xu Q, Qi P, Tan C, et al: PTTG3P promotes gastric tumour cell proliferation and invasion and is an indicator of poor prognosis. $\mathrm{J}$ Cell Mol Med 21: 3360-3371, 2017.

14. Tang Z, Li C, Kang B, Gao G, Li C and Zhang Z: GEPIA: A web server for cancer and normal gene expression profiling and interactive analyses. Nucleic Acids Res 45 (W1): W98-W102, 2017.

15. Chandrashekar DS, Bashel B, Balasubramanya SAH Creighton CJ, Ponce-Rodriguez I, Chakravarthi BVSK and Varambally S: UALCAN: A portal for facilitating tumor subgroup gene expression and survival analyses. Neoplasia 19: 649-658, 2017.

16. Rhodes DR, Kalyana-Sundaram S, Mahavisno V, Varambally R, $\mathrm{Yu}$ J, Briggs BB, Barrette TR, Anstet MJ, Kincead-Beal C, Kulkarni P, et al: Oncomine 3.0: Genes, pathways, and networks in a collection of 18,000 cancer gene expression profiles. Neoplasia 9: 166-180, 2007.

17. Rhodes DR, Yu J, Shanker K, Deshpande N, Varambally R, Ghosh D, Barrette T, Pandey A and Chinnaiyan AM ONCOMINE: A cancer microarray database and integrated data-mining platform. Neoplasia 6: 1-6, 2004.

18. Hou GX, Liu P, Yang J and Wen S: Mining expression and prognosis of topoisomerase isoforms in non-small-cell lung cancer by using Oncomine and Kaplan-Meier plotter. PLoS One 12: e0174515, 2017.

19. Lou W, Chen J, Ding B, Chen D, Zheng H, Jiang D, Xu L, Bao C Cao $\mathrm{G}$ and Fan W: Identification of invasion-metastasis-associated microRNAs in hepatocellular carcinoma based on bioinformatic analysis and experimental validation. J Transl Med 16: 266, 2018

20. Lou W, Liu J, Ding B, Chen D, Xu L, Ding J, Jiang D, Zhou L, Zheng S and Fan W: Identification of potential miRNA-mRNA regulatory network contributing to pathogenesis of HBV-related HCC. J Transl Med 17: 7, 2019.

21. Győrffy B, Surowiak P, Budczies J and Lánczky A: Online survival analysis software to assess the prognostic value of biomarkers using transcriptomic data in non-small-cell lung cancer. PLoS One 8: e82241, 2013.

22. Fuertes M, Sapochnik M, Tedesco L, Senin S, Attorresi A, Ajler P, Carrizo G, Cervio A, Sevlever G, Bonfiglio JJ, et al: Protein stabilization by RSUME accounts for PTTG pituitary tumor abundance and oncogenicity. Endocr Relat Cancer 25: 665-676, 2018.
23. Read ML, Fong JC, Modasia B, Fletcher A, Imruetaicharoenchoke W, Thompson RJ, Nieto H, Reynolds JJ, Bacon A, Mallick U, et al: Elevated PTTG and PBF predicts poor patient outcome and modulates DNA damage response genes in thyroid cancer. Oncogene 36: 5296-5308, 2017.

24. Gao H, Zhong F, Xie J, Peng J and Han Z: PTTG promotes invasion in human breast cancer cell line by upregulating EMMPRIN via FAK/Akt/mTOR signaling. Am J Cancer Res 6: 425-439, 2016.

25. Wang X, Duan W, Li X, Liu J, Li D, Ye L, Qian L, Yang A, Xu Q, Liu H, et al: PTTG regulates the metabolic switch of ovarian cancer cells via the c-myc pathway. Oncotarget 6: 40959-40969, 2015.

26. Kakar SS and Malik MT: Suppression of lung cancer with siRNA targeting PTTG. Int J Oncol 29: 387-395, 2006.

27. Mu YM, Oba K, Yanase T, Ito T, Ashida K, Goto K, Morinaga H, Ikuyama S, Takayanagi R and Nawata H: Human pituitary tumor transforming gene (hPTTG) inhibits human lung cancer A549 cell growth through activation of p21(WAF1/CIP1). Endocr J 50: 771-781, 2003.

28. Rehfeld N, Geddert H, Atamna A, Rohrbeck A, Garcia G, Kliszewski S, Neukirchen J, Bruns I, Steidl U, Fenk R, et al: The influence of the pituitary tumor transforming gene-1 (PTTG-1) on survival of patients with small cell lung cancer and non-small cell lung cancer. J Carcinog 5: 4, 2006.

29. Shah PP, Fong MY and Kakar SS: PTTG induces EMT through integrin $\alpha \mathrm{V} \beta 3$-focal adhesion kinase signaling in lung cancer cells. Oncogene 31: 3124-3135, 2012.

30. Honda S, Hayashi M, Kobayashi Y, Ishikawa Y, Nakagawa K and Tsuchiya E: A role for the pituitary tumor-transforming gene in the genesis and progression of non-small cell lung carcinomas. Anticancer Res 23: 3775-3782, 2003. International (CC BY-NC-ND 4.0) License. 